\title{
25 Graduate Student Award Finalists Participate in Special Session at MRS Fall Meeting
}

Twenty-five graduate student award finalists are participating in a special student talk session during the 1989 MRS Fall Meeting in Boston. A recent addition to the criteria for selecting award recipients, this special session is being implemented at the Fall Meeting to reflect the growing competitiveness of the graduate student awards program, to improve the selection process, and to give more visibility to the graduate students at the meeting.

The finalists are presenting 10-minute talks on their work Tuesday, November 28 from 12:10 to 1:30 p.m. in five designated rooms at the Boston Marriott Hotel. Among the judges will be organizers from the symposia in which the finalists are participating, members of the MRS Awards Committee, and other MRS representatives. MRS Fall Meeting participants are also invited to attend.

Graduate student awards will be presented at the Von Hippel Award Ceremony, Wednesday, November 29 at 6 p.m. in the Boston Marriott Hotel. Following is a list of the finalists, the titles of their papers, and the symposia in which the complete papers are being presented.

\section{Graduate Student Award Finalists 1989 MRS Fall Meeting}

Terry Lynn Alford, Cornell University, "Ion-Beam Synthesis of Buried Yttrium Silicide," (Symposium A)

John F. Ankner, University of Illinois, "Neutron Reflectivity Study of GD-Y Interface," (Symposium J)

Louis Breaux, University of Texas, "Remote Plasma-Enhanced CVD of Epitaxial Silicon on Silicon (100) at $150^{\circ} \mathrm{C}^{\prime \prime}$ (Symposium I)

David Ward Brown, University of California-Davis, "Formation of Buried Elemental Layers Using Ion Implantation," (Symposium A)
Ju Ren Ding, Tsinghua University, Beijing, "Ion Beam Method to Study Fractal Aggregation of Magnetic Particles in Thin Films," (Symposium A)

Vinayak P. Dravid, Lehigh University, "Plan-View CBED Studies of NiO$\mathrm{ZrO}_{2}(\mathrm{CaO})$ Interfaces," (Symposium $\mathrm{C}$ )

Hailing Duan, John Hopkins University, "Studies on the Formation of Switching and Memory Storage Materials Constructed from Metallo-Organic ChargeTransfer Salts," (Symposium Q)

Hilary L. Hampsch, Northwestern University, "Second Order Nonlinear Optics and Polymer Physics of Corona Poled Polymer Films," (Symposium Q)

Arun Inam, Rutgers University, "Josephson Weak-Links Fabricated from Heteroepitaxial $\mathrm{YBa}_{2} \mathrm{Cu}_{3} \mathrm{O}_{7-\mathrm{x}} / \mathrm{PrBa}_{2} \mathrm{Cu}_{3} \mathrm{O}_{7-x} /$ $\mathrm{YBa}_{2} \mathrm{Cu}_{3} \mathrm{O}_{7 x}$ Multilayers," (Symposium M)

Forrest Kaatz, University of Pennsylvania, "Epitaxial Growth of $\mathrm{TbSi}_{2}$ on $\mathrm{Si}(111)$," (Symposium D)

Robert R. Keller, University of Minnesota, "Electron Channeling Analysis of Elastic Strains in InGaAs Thin Films," (Symposium D)

Qiyuan Ma, Columbia University, "Use of Si-YBaCuO Intermixed System for Patterning of Superconducting Thin Films," (Symposium M)

John F. Marko, Massachusetts Institute of Technology, "Weighted-Density Theory of Phase Transitions in Fluids Composed of Anisotropic Particles," (Symposium V)

Anne W. Mayes, Northwestern University, "Microphase Separation in Multiblock Copolymer Melts," (Symposium S)

Rejean Munger, University of Waterloo, Ontario, "Partial Superconductivity in $\mathrm{YBa}_{2} \mathrm{Cu} 3 \mathrm{O}_{7}$ at $90<\mathrm{T}<300 \mathrm{~K}$," (Symposium M)

Linda S. Sapochak, University of South- ern California, "Effects of Conjugation Length and Substituents on the Conductivity of Oxidatively Doped a,wDiphenylpolenes," (Symposium Q)

Linda S. Schadler, University of Pennsylvania, "Interphase Behavior of Cyclic Fatigue of Monofilament Composites," (Symposium N)

Xiangdong Shi, University of Pennsylvania, "Sound Velocity Studies of the Bechgaard Salts (TMTSF) ${ }_{2} \mathrm{ClO}_{4}$ and (TMTSF) ${ }_{2} \mathrm{PF}_{6}$," (Symposium Q)

Rajiv K. Singh, North Carolina State University, "Pulsed Laser Technique for Deposition of Superconducting Thin Films: Deposition Physics and In-Situ Processing," (Symposium M)

Leland Scott Swanson, Iowa State University, "Photoluminescence, ESR, and ODMR Studies of Pristine and Photodegraded Poly(3-Hexylthiopohene) Films and Solutions," (Symposium Q)

Igor Szafranek, University of Illinois at Urbana-Champaign, "Reassessment of Acceptor Passivation Mechanism in pType Hydrogenated GaAs," (Symposium G)

Lisa Tietz, Cornell University, "The Structure of Interfaces in Oxide Heterojunctions Formed by CVD," (Symposium C)

Karen I. Winey, University of Massachusetts, "The Ordered Bicontinuous Double Diamond Structure in Blends of Diblock Copolymer and Homopolymer," (Symposium $\mathrm{O}$ )

Dan Q. Wu, State University of New York at Stony Brook, "Small-Angle X-Ray Scattering on Poly(Ethylene-Methacrylic Acid) Lead and Lead Sulfide Ionomers," (Symposium O)

Satoshi Yamauchi, Tohoku University, Japan, "Plasma-Assisted Epitaxial Growth of ZnSe Films in Hydrogen Plasma," (Symposium E)

\section{Do You Have An Opinion?}

The MRS BULLETIN wants your comments and views on issues affecting materials research.

Send your comments to: Editor, MRS BULLETIN, 9800 McKnight Road, Pittsburgh, PA 15237

Telephone (412) 367-3036, Fax (412) 367-4373 


\section{Essential}

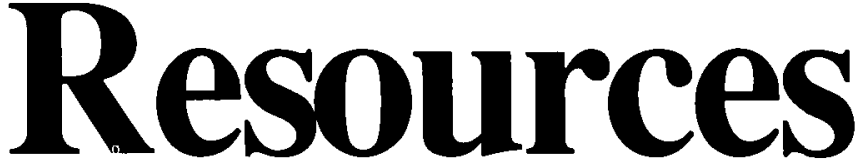

\section{Visit booth \#114 in Boston}

\section{Organometallic Vapor- Phase Epitaxy \\ Theory and Practice Gerald B. Stringfellow}

Here is the first single-author treatment of organometallic vapor-phase epitaxy (OMVPE) - a leading technique for the fabrication of semiconductor materials and devices. Also included are metalorganic molecular-beam epitaxy (MOMBE) and chemical-beam epitaxy (CBE) ultra-high-vacuum deposition techniques using organometallic source molecules. This book provides a basic foundation for understanding the technique and the application of OMVPE for the growth of both III-V and II-VI semiconductor materials and the special structures required for device applications.

July 1989,416 pages, $\$ 59.50$

ISBN: 0-12-673840-8

\section{Electronic Materials}

Science and Technology

Shyam P. Murarka and

Martin C. Peckerar

Written as an intermediate-level text, this book will also be a good reference source for practicing engineers and scientists who are interested in electronic materials and devices. After presenting an introduction to electronic devices, the authors describe the processing of a complementary MOS (CMOS) circuit including fundamental physical and materials concepts. Other areas of electronic material processing covered include crystal growth, oxidation, diffusion, ion implantation, metallization, chemical vapor deposition, lithography concepts and tools, and etching.

September 1989,640 pages, $\$ 59.95$

ISBN: 0-12-511120-7

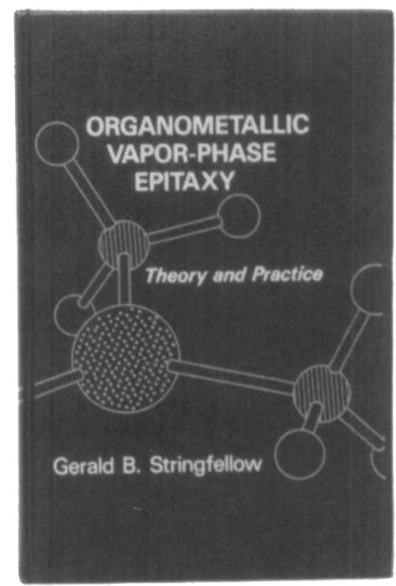

\section{Aluminum Alloys- Contemporary Research and Applications \\ edited by \\ A.K. Vasudevan and R.D. Doherty}

This book discusses the structure and properties of the current and potential aluminum alloys in terms of their structure (and structural transformations by new processing methods) and the relationship between structure and mechanical and other properties. The alternative materials that challenge aluminum are considered as well, since the challenge of new competitive materials is a strong influence on innovation.

October 1989 , c. 678 pages, $\$ 149.00$

ISBN: 0-12-341831-3

\section{Plasma Etching}

An Introduction

edited by

Dennis M. Manos and Daniel L. Flamm

Plasma etching plays an essential role in microelectronic circuit manufacturing. Suitable for researchers, process engineers, and graduate students, this book introduces the basic physics and chemistry of electrical discharges and relates them to plasma etching mechanisms. Throughout the volume the authors offer practical examples of process chemistry, equipment design, and production methods.

July 1989,496 pages, $\$ 69.50$

ISBN: 0-12-469370-9

\section{Nanostructure Physics and Fabrication}

Proceedings of the International Symposium, College Station, Texas, March 13-15, 1989 edited by

Mark A. Reed and Wiley P. Kirk

Nanostructure Physics and Fabrication contains the contributions of an interdisciplinary group of specialists in nanometer scale fabrication, physics of mesoscopic systems, electronic transport, and materials science brought together to discuss the current status of nanometer scale electronic structures. These articles provide the most current assessment of this active and growing area of interest.

CONTENTS: Overview. Conceptual Origins of Nanostructures. Lateral Periodicity and Confinement. Quantum Devices and Transistors. Equilibrium and Nonequilibrium Response in Nanoelectronic Structures. Quantum Wires and Ballistic Point Contacts. Related Fabrication and Phenomena.

August 1989,544 pages, $\$ 64.50$ ISBN: 0-12-585000-X

Please visit Booth No. 114 at the MRS Show in Boston, November 28-30, 1989.

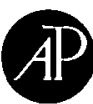

ACADEMIC PRESS Harcourt Brace Jovanovich, Publishers Book Marketing Department \#18119 1250 Sixth Avenue, San Diego, CA 92101

CALL TOLL FREE

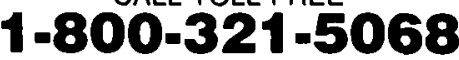

Quote this reference number for free postage and handling on your prepaid order $\mathbf{- 1 8 1 1 9}$

Prices subjoct to change without notice. D1989 by Academic Press, Inc.
All Rights Resereved. DK/KR - 18119 . 


\title{
1989 MRS Fall Meeting Symposium Proceedings and Extended Abstracts
}

\author{
Available at Special Pre-Publication Prices Through March 1, 1990!
}

\author{
Beam-Solid Interactions: Physical Phenomena \\ Editors: J.A. Knapp, P. Borgesen, R.A. Zuhr \\ Order Code 157B \\ $\$ 32.00$ MRS Member \\ $\$ 44.00$ U.S. List $\$ 50.00$ Foreign \\ In-Situ Patterning: Selective Area Deposition and \\ Etching \\ Editors: R. Rosenberg, A.F. Bernhardt, J.G. Black \\ Order Code $158 \mathrm{~B}$ \\ $\$ 34.00$ MRS Member \\ $\$ 39.00$ U.S. List $\$ 45.00$ Foreign \\ Atomic Scale Structure of Interfaces \\ Editors: R.D. Bringans, R.M. Feenstra, J.M. Gibson \\ Order Code 159B \\ \$34.00 MRS Member \\ $\$ 39.00$ U.S. List $\$ 44.00$ foreign
}

Layered Structures: Heteroepitaxy, Superlattices,

Strain, and Metastability

Editors: B.W. Dodson, L.J. Schowalter,

J.E. Cunningham, F.H. Pollak

Order Code $160 \mathrm{~B}$

$\$ 34.00$ MRS Member

$\$ 39.00$ U.S. List $\$ 44.00$ Foreign

Properties of II.VI Semiconductors: Bulk Crystals, Thin Films, Quantum Well Structures and Dilute Magnetic Systems

Editors: J.F. Schetina, F.J. Bartoli Jr., H.F. Schaake

Order Code 1618

\$34.00 MRS Member

$\$ 39.00$ U.S. List $\$ 44.00$ Foreign

Diamond, Boron Nitride, Silicon Carbide and

Related Wide Bandgap Semiconductors

Editors: J.T. Glass, R.F. Messier, N. Fujimori

Order Code 162B

$\$ 32.00$ MRS Member

$\$ 36.00$ U.S. List \$41.00 Foreign

Impurities, Defects and Diffusion in Semiconduc-

tors: Bulk and Layered Structures

Editors: J. Bernholc, E.E. Haller, D.J. Wolford

Order Code 163B

$\$ 42.00$ MRS Member

$\$ 48.00$ U.S. List $\$ 55.00$ Foreign

Materials Issues in Microcrystalline

Semiconductors

Editors: P.M. Fauchet, C.C. Tsai, K. Tanaka

Order Code 164B

$\$ 36.00$ MRS Member

$\$ 41.00$ U.S. List $\$ 47.00$ Foreign

Characterization of Plasma-Enhanced

CVD Processes

Editors: G. Lucousky, D.E. Ibbotson, D.W. Hess

Order Code 165B

$\$ 26.00$ MRS Member

$\$ 31.00$ U.S. List $\$ 36.00$ Foreign
Neutron Scattering for Materials Science

Editors: S.M. Shapiro, S.C. Moss, J.D. Jorgensen

Order Code 1668

$\$ 36.00$ MAS Member

$\$ 41.00$ U.S. List \$47.00 Foreign

Advanced Electronic Packaging Materials

Editors: J. Partridge, C-Y. Li, C.J. Chen, A. Barfknecht

Order Code 1678

$\$ 30.00$ MRS Member

$\$ 35.00$ U.S. List $\$ 42.00$ Foreign

Chemical Vapor Deposition of Refractory Metals and Ceramics

Editors: T.M. Besmann, B.M. Gallois

Order Code 1688

$\$ 30.00$ MRS Member

$\$ 35.00$ U.S. List $\$ 40.00$ Foreign

High Temperature Superconductors: Fundamental

Properties and Novel Materials Processing

Editors: J. Narayan, C.W. Chu, L.F. Schneemeyer,

D.K. Christen

Order Code 1698

$\$ 38.00$ MRS Member

$\$ 43.00$ U.S. List $\$ 49.00$ Foreign

Tailored Interfaces in Composite Materials

Editors: C.G. Pantano, E.J.H. Chen

Order Code 170B

\$34.00 MRS Member

$\$ 39.00$ U.S. List $\$ 46.00$ Foreign

Polymer Based Molecular Composites

Editors: D.W. Schaefer, J.E. Mark

Order Code 1718

$\$ 34.00$ MRS Member

$\$ 39.00$ U.S. List \$46.00 Foreign

Optical Fiber Materials and Processing

Editors: J.W. Feming, G.H. Sigel, S. Takahashi,

P.W. France

Order Code 1728

$\$ 28.00$ MRS Member

$\$ 34.00$ U.S. List $\$ 39.00$ Foreign

Electrical, Optical and Magnetic Properties of

Organic Solid-State Materials

Editors: L.Y. Chiang, D.O. Cowan, P. Chaikin

Order Code 1738

$\$ 36.00$ MRS Member

$\$ 41.00$ U.S. List \$47.00 Foreign

Materials Synthesis Utilizing Biological Processes

Editors: P.D. Calvert, M. Alper, P.C. Rieke

Order Code 1748

$\$ 26.00$ MRS Member

$\$ 29.00$ U.S. List $\$ 33.00$ Foreign
Multi-Functional Materials

Editors: D.R. Urich, FE. Karasz, A.J. Buckley,

G. Gallagher-Daggitt

Order Code 175B

\$26.00 MRS Member

$\$ 30.00$ U.S. List $\$ 35.00$ Foreign

Fractal Aspects of Materials

Editors: J.E. Martin, J.H. Kaufman, P.W. Schmidt

Order Code EA-2OB

$\$ 18.00$ MRS Member

$\$ 20.00$ U.S. List $\$ 22.00$ Foreign

Scientific Basis for Nuclear Waste

Management XIII

Editors: V.M. Oversby, PW. Brown

Order Code 176B

$\$ 45.00$ MRS Member

$\$ 50.00$ U.S. List $\$ 55.00$ Foreign

Macromolecular Liquids

Editors: C.R. Safinya, S.A. Safran, P.A. Pincus

Order Code $177 \mathrm{~B}$

$\$ 36.00$ MRS Member

$\$ 41.00$ U.S. List $\$ 47.00$ Foreign

Fly Ash and Coal Conversion By-Products:

Characterization, Utilization and Disposal VI

Editors: R.L. Day, F.P. Glasser

Order Code 1788

$\$ 32.00$ MRS Member

$\$ 37.00$ U.S. List \$42.00 Foreign

Specialty Cements with Advanced Properties Editors: H. Jennings, A.G. Landers, B.E. Scheetz,

I. Odler

Order Code १९९

\$30.00 MRS Member

$\$ 35.00$ U.S. List $\$ 42.00$ foreign

MRS accepts check or money order Ipayable in U.S. Dollarsl, purchase order, and Visa, MasterCard and Diners Club cards. Order from the Materials Research Society, Publications Department, 9800 McKnight Road, Pittsburgh, PA 15237; telephone |412| 3673012; fax (412) 367-4373. In Europe, Africa and the Middle East order from Clarke Associates-Europe Ltd., 13a Small Street, Bristol BS1 10E, England; telephone: 0272 268864; fax: 0272226437.

The prices listed here are available only through March 1, 1990. Prices after this date will be higher. Order now and savel 


\section{EQUIPMENT EXHIBIT at the 1989 MRS Fall Meeting}

As part of the 1989 MRS Fall Meeting in Boston, a major equipment exhibit will display analytical and processing equipment closely paralleling the nature of the technical symposia and short courses. The exhibit will be conveniently located on thee third floor of the Boston Marriott Hotel/Copley Place.

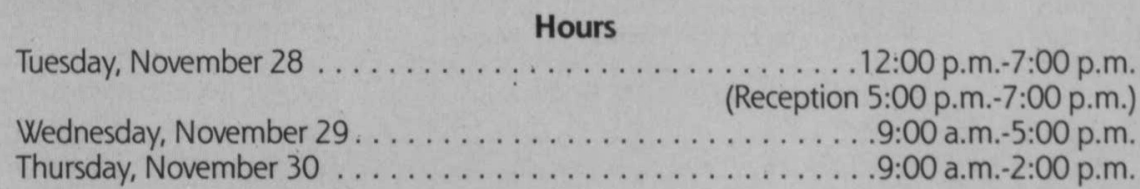

\section{Exhibitors \\ (as of September 13, 1989)}

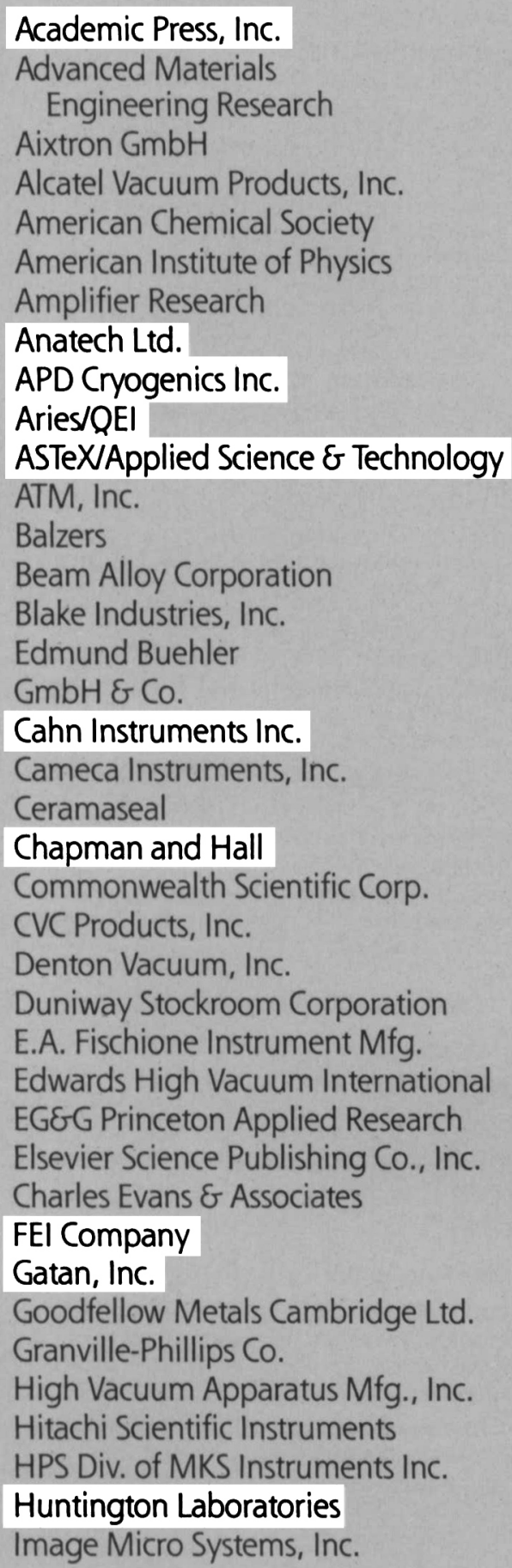

Implant Sciences Corporation

Inel Inc.

Innovative Technology, Inc.

Instruments SA, Inc.

International Scientific Instruments

Ion Tech, Inc.

Janis Research Company

JCPDS-ICDD

JEOL U.S.A., Inc.

Kaiser Systems, Inc.

Keithley Instruments

Kevex Instruments, Inc.

Kimball Physics, Inc.

Kratos Analytical, Inc.

Lake Shore Cryotronics, Inc.

Lambda Physik, Inc.

Lehighton Electronics, Inc.

Kurt J. Lesker Company

Leybold Inficon

Leybold Vacuum Products

Materials by Metron

McAllister Technical Services

MDC Vacuum Products Corporation

Microscience, Inc.

MKS Instruments Inc.

MMR Technologies, Inc.

National Electrostatics Corp.

Neslab Instruments

Netzsch Incorporated

NGS Associates, Inc.

Nicolet

North Eastern Analytical Corp.

Omicron Associates

Oxford Instruments

Oxford University Press

Peabody Scientific

Pergamon Press

Perkin-Elmer Corporation

Philips Electronic Instruments Co.
Physicon Corporation

Plasma Technology

Plenum Publishing Corporation

Princeton Gamma-Tech, Inc.

Princeton Instruments

Process Products Corporation

Quantachrome Corp.

Quantum Design, Inc.

RHK Technology, Inc.

Rigaku/USA, Inc.

RMC-Cryosystems Inc.

Rudolph Research

Scintag, Inc.

Siemens Analytical X-Ray

South Bay Technology, Inc.

Spectramass, Inc.I

Spectra Instruments Div.

Spectra-Tech Inc.

Spire Corporation

Springer-Verlag New York Inc.

Structure Probe, Inc./SPI Supplies

Sula Technologies

Superconductive Components, Inc.

Sycon Instruments, Inc.

Tencor Instruments

Thermionics Laboratory

Ultra High Vacuum Instruments, Inc.

Vacuum Barrier Corp.

Varian/Vacuum Products

VAT, Inc.

VCR Group, Inc.

VG Instruments, Inc.

Virginia Semiconductor, Inc.

Voltaix, Inc.

Walff Associates

Waterloo Scientific

Wavemat, Inc.

John Wiley \& Sons

Carl Zeiss, Inc. 


\section{0}

\section{SPRING \\ MEETING}

\section{PROGRAM}

$\mid \begin{aligned} & \text { April 16-21, } \\ & 1990\end{aligned}$

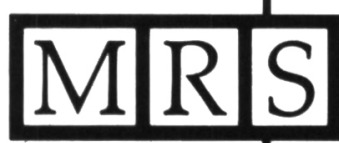

MATERIALS RESEARCH SOCIETY

BETTER CERAMICS THROUGH CHEMISTRYIV

C.J. Brinker, Sandia National Laboratories, (505) 846-3552, FAX (505) 846-5064: D.E. Clark. University of Florida, (904) 392-7660. FAX (904) 392-6359: Donald R. Ulirich. Air Force Office of Scientific Re search. (202) 767-4963; Brian J.J. Zelinski, Arizona Materials Laboratories, (602) 322-2977. FAX (602) $322 \cdot 2993$

\section{ADVANCED METALLIZATIONS IN}

MICROELECTRONICS

Avishay Katz, AT\&T Bell Laboratories, (201) 582-2261, FAX (201) 582-5917; Shyam P. Murarka, Rensselaer Polytechnic Institute, (518) 276-2978. FAX [518) 2768761: Ami Appelbaum, Rockwell International Corporation, (214) 996-6522, FAX (214) 996-5545

\section{POLYSILICON THIN FILMS AND INTERFACES}

Bruha Raicu, Integrated Technology Associate, (408) 773-8614: FAX (415) 941-2704: T. Kamins, Hewlett-Packard, (415) 857-5470, FAX (415) 8575308: Carl V. Thompson. Massachusetts Institute of Technology. (617) 253-7652, FAX (617) 258-8539

\section{CRITICAL CURRENTS IN HIGH-TEMPERATURE}

SUPERCONDUCTORS

John R. Clem. lowa State University, (515) 294-4223. FAX (515) 294-0689: Jack W. Ekin, National Institute of Standards \& Technology, (303) 497-5448, FAX (303) 497-5316; Sungho Jin. AT\&T Bell Laboratories, (201) 582-4076, FAX (201) 582-2913; Donald M. Kroeger, Oak Ridge National Laboratory, (615) 574 . 5155. FAX (615) 574-6073

HIGH RESOLUTION ELECTRON MICROSCOPY OF DEFECTS IN MATERIALS

Robert Sinclair, Stanford University, (415) 723-1102, FAX (415) 725-4034; Ulrich Dahmen, University of California, Berkeley, (415) 486-4627. FAX (415) 4864888; David J. Smith. Arizona State University, (602) $965-4540$
DEGRADATIONMECHANISMSINIII-V COMPOUND SEMICONDUCTOR DEVICES \& STRUCTURES

V. Swaminathan. AT\&T Bell Laboratories. (201) 582 4981. FAX (201) 582-5917; Stephen J. Pearton, AT\& Bell Laboratories, (201) 582-4757, FAX (201) 582 5917: Omar Manasreh, Wright Research \& Devel opment Center, (513) 255-4474, FAX (513) 255-5375

\section{MATERIALS ISSUES IN ART AND} ARCHAEOLOGY II

James R. Druzik, Getty Conservation Institute, (213) 822-2299: FAX (213) 821-9409; Pamela B. Vandiver Smithsonian Institution (301) 238-3734; FAX (301) 2383667. George Wheeler, Metropolitan Museum of Art, (212) 570-3858. FAX (212) 570-3879

MATERIALS FOR SENSORS AND SEPARATIONS

Marc Anderson, University of Wisconsin-Madison (608) 262 2470. FAX (608) 262-0454; John Armor, Air Products and Chemicals, Inc. (215) 481-5792, FAX (215) 481-4600: D. Jed Harrison University of Alberta, (403) 492 2790, FAX (403) 492-8231: Antonio J. Ricco Sandia Nationa Laboratories, (505) 846-4947 FAX (505) 846-2009

\section{ALLOY PHASE STABILITY AND} DESIGN

G. Malcolm Stocks, Oak Ridge National Laboratory. (615) 574 . 5163: Anthony F. Giamei, United Technologies Research Center David P. Pope. University of Pennsylvania, (215) 898-9837. FAX (215) $898-1130$

THIN FILM STRUCTURES AND PHASE STABILITY

Bruce M. Clemens, Stanford University, (415) 725-7455, FAX (415) 725-4034. William L. Johnson. California Institute of Technology. (818) 356-4433, FAX (818) $795-1547$

\section{THIN FILMS: STRESSES AND MECHANICAL}

PROPERTIES II

Warren Oliver, Oak Ridge National Laboratory, (615) 576-7245, FAX (615) 574-7721: Mary Doerner, International Business Machines, (408) 284-8369, FAX (408) 256-8481: George Pharr, Rice University, (713) 527-8101, Ext. 3573, FAX (713) 285-5136, Bitnet: PHARR@RLCE; Franz R. Brotzen, Rice University. (713) 527-8101, FAX (713) 285-5136

MICROWAVE PROCESSING OF MATERIALS

William B. Snyder, Oak Ridge National Laboratory, (615) 576-2178. Willard H. Sutton, United Technologies Research Center (203) 727-7639: D Lynn Johnson, Northwestern University, (312) 491-3584 Magdy F. Iskander: University of Utan, (801) 581

\section{PLASMA PROCESSING AND SYNTHESIS OF}

MATERIALS

Diran Apelian, Drexel University, (215) 895-1541, FAX (215) 895-4929: Julian Szekely. Massachusetts Institute of Technology, (617) 253-3236; FAX (617) 2538124

ASER ABLATION FOR MATERIALS SYNTHESIS

David C. Paine. Brown University, (401) 863-1457. FAX (401) 863-1157: John C. Bravman, Stanford University, (415) 723-3698, FAX (415) 725-4034

\section{AMPHOUS SILCON TECHNOLOGY - 1990}

P. Taylor University of Utah (801) 581-4484, FAX (801) 581-4801: Malcolm J. Thompson, Xerox PARC (415) 494-4561. FAX (415) 494-4919; Y. Hamakawa, (4) 853-1362: Arun Madan, Colorado, (303) 526-9016 FAX (303) 526-1718: P.G. LeComber, University of Dundee, United Kingdom, 44-382-23181, FAX 44382-201604
SURFACE AND NEAR SURFACE STRUCTURE OF POLYMER INTERFACES

Jeffry A. Kelber, Sandia National Laboratories. (505) 844-5436; Ralph G. Nuzzo. AT\&T Bell Laboratories, (201) 582-5486: Matthew V. Tirrell, University of Minnesota, (612) 625-0192, FAX (612) 626-7246: Ernesto Occhiello, Istituto Guido Donegani, Italy ATOMIC SCALE CALCULATIONS OF STRUCTURE IN MATERIALS

Michael A. Schluter AT\&T Bell Laboratories, (201) 582-3106: Murray S. Daw, Sandia National Laboratories. (415) 294-2198

\section{INTERMETALLIC MATRIX COMPOSITES}

Donald L. Anton, United Technologies Research Center, (203) 727-7174, FAX (203) 727-7879; Robert McMeeking, University of California, Santa Bar. bara, (805) 961-4583. FAX (805) 961-8124: Daniel Miracle United States Air Force Wright-Patterson AFB. (513) 255-9833, FAX (513) 255-9792; Patrick Martin, Los Alamos National Laboratory, (505) 667 8168, FAX (505) 667-1754

PHYSICAL PHENOMENA IN GRANULAR MATERIALS Theodore H. Geballe. Stanford University. (415) 723 0215, FAX (415) 723-0010; Ping Sheng. Exxon Research \& Engineering, (201) 730-2870, FAX (201) 730-3042: G.D. Cody, Exxon Research \& Engineering. (201) 730-3022; FAX (201) 730-3042

\section{SUPERPLASTICITY IN METALS, CERAMICS, AND} INTERMETALLICS

Merrilea J. Mayo, Sandia National Laboratories, (505) 846-3551, FAX (505) 846-5064: Jeffrey Wadsworth, Lockheed Missile \& Space Co., Inc.. (415) 424-2234. FAX (415) 354-5415; Masaru Kobayashi. Technological University of Nagaoka, Japan, 0258-46-6000, Ext. 7120, FAX 0258-46-6972; Amiya K. Mukherjee. University of California at Davis, (916) 752-1776: FAX (916) 752-8058

MATERIALS INTERACTIONS RELEVANT TO THE PULP, PAPER AND WOOD INDUSTRIES

June D. Passaretti, Pfizer Minerals Research Center, (215) 861-3431, FAX (215) 861-3412; Daniel Caulfield. USDA Forest Service, (608) 231-9436. FAX (608) 231. 9592: Rustum Roy, Pennsylvania State University, (814) 865-3421: FAX (814) 865.2326: Vance Setterholm, USDA Forest Service, (608) 231.9478: FAX (608) 231-9592

EPITAXIAL HETEROSTRUCTURES

Don W. Shaw, Texas Instruments, Inc., (214) 9954788. FAX (214) 995-5539: John C. Bean, AT\&T Bell Laboratories, (201) 582-3324, FAX (201) 582-3901. Vassilis G. Keramidas, Bellcore, (201) 758-3353, FAX (201) 758-9626: Paul S. Peercy, Sandia National Laboratories, (505) 844-4309. FAX (505) 846-2009

WORKSHOP ON SPECIMEN PREPARATION FOR TRANSMISSION ELECTRON MICROSCOPY OF MATERIALS II

Ron Anderson, IBM, (914) 892-2225, FAX (914) 8922555

FRONTIERS OF MATERIALS RESEARCH

Robert A. Huggins. Stanford University, (415) 723. 4110. FAX (415) 725-4034

\section{FERROELECTRIC THIN FILMS}

Angus I. Kingon, North Carolina State University, (919) 737-2347. FAX (919) 737-3419: Edward R Myers, National Semiconductor, (408) 721-2258. FAX (408) 736.8503

\section{Meeting Chairs}

John C. Bravman

Stanford University

(415) 723-3698

FAX (415) 725-4034

C. Jeffrey Brinker

Sandia National

Laboratories

(505) 846-3552

FAX (505) 846-5064
William H. Butler

Oak Ridge

National Laboratory (615) 574-4845

FAX (615) 574-7721 
- SYMPOSIUM A / SURFACE CHEMISTRY AND BEAM-SOLID INTERACTIONS Harry A. Atwater, Caliech, (818) 356-2197 Frances A. Houle, IBM Almaden Research Center, (408) $927-2420$

Doug Lowndes, Oak Ridge National Laboratory, (615) 5746306

- SYMPOSIUM B / ELECTRONIC, OPTICAL AND DEVICE PROPERTIES OF LAYERED STRUCTURES

John Hayes, Belloore, (201) 758-2851

Mark Hybertsen, AT\&T Bell Laboratories,

(201) 582.3628

Eicke Weber, University of Calitornia, (415) 642-0205, FAX (415) 486-5933

- SYMPOSIUM C I MICROSTRUCTURAL EVOLUTION OF SURFACES AND THIN FILMS

Carl V. Thompson, Massachusetts Institute of Technology (617) 253.7652

Jeffrey Y. Tsao, Sandia National Laboratories, (505) 8447092

David Srolovitz, University of Michigan, (313) 936-1740

- SYMPOSIUM D / ELECTRONIC PACKAGING MATERIALS SCIENCE

Edwin D. Lillie, MCC, (512) 250-2715

Kenneth A. Jackson, AT\&T Bell Laboratories, (201) 582.4188

Ralph J. Jaccodine, Lehigh University, (215) 758-4409

- SYMPOSIUM E / CHEMICAL PERSPECTIVES OF MICROELECTRONIC

MATERIALS

Minal E. Gross, AT\&T Bell Laboratories, (201) 582-4504 Lawrence H. Dubois, AT\&T Bell Laboratories, (201) $582-7920$

Leonard V. Interrante, Rensselaer Polytechnic Institute, (518) 276-2644

Klaus F. Jensen, Massachusetts Institute of Technology, (617) $253-4589$

\section{- SYMPOSIUM F / PHASE TRANSFOR-}

MATIONS

Michael O. Thompson, Cornell University, (607) 255-4714

- SYMPOSIUM G / CLUSTERS \& CLUSTER-ASSEMBLED MATERIALS Robert S. Averback, University of Illinois-Urbana, (217) $333-4302$

David L. Nelson, Office of Naval Research, (202) $696-4410$

J. Bernholc, North Carolina State University (919) $737-3126$

\section{- SYMPOSIUM H / HIGH-TEMPERATURE} SUPERCONDUCTORS

Kenneth Lay, GE Corporate Research and

Development Center, (518) 387.7495

Julia M. Phillips, AT\&T Bell Laboratories,

(201) $582-4428$

Allen Goldman, University of Minnesota,

(612) 624-6525

Anthony C. Schaffhauser, Oak Ridge National

Laboratory, (615) 574-4826
- SYMPOSIUM I / MECHANICAL PROPERTIES OF POROUS MATERIALS Lorna J. Gibson, Massachusetts Institute of Technology, (617) 253-7107

Karl Sieradzki, The Johns Hopkins University, (301) $338-5409$

David Green, Pennsylvania State University, (814) $863-2011$

\section{- SYMPOSIUM J / ADVANCED DIFFRAC-} TION METHODS

Philip I. Cohen, University of Minnesota, (612) 625-5517 David Eaglesham, AT\&T Bell Laboratories, (201) $582-3768$

Ting C. Huang, IBM Almaden Research Center (408) $927-2375$

\section{- SYMPOSIUM K/ DEFECTS IN}

MATERIALS

Paul D. Bristowe, Massachusetts Institute of

Technology, (617) 253-3326

Ernst Epperson, Argonne National Laboratory, (312) $972-4971$

J.E. Griffith, AT\&T Bell Laboratories, (201) 582-5222

Z. Lillienta-Weber, University of California-Berkeley, (415) $486-6276$

\section{- SYMPOSIUM L / SOLID STATE IONICS} Gholamabbas Nazri, GM Research Laboratory (313) $986-0737$

Duward F. Shriver, Northwestern University, (312) $491-5655$

M. Balkanski, Universite Pierre et M. Curie, France Robert A. Huggins, Stanford University, (415) 723-4110; FAX (415) 725-4034

\section{- SYMPOSIUM M / KINETICS IN SMALL} CONFINING SYSTEMS

J.M. Drake, Exxon Research and Engineering, (201) $730-2848$

R. Kopelman, University of Michigan, (313) 7647541 J. Klafter, Tel Aviv University, Israel, 972-3-5450254, FAX 972-3-541-3752

\section{- SYMPOSIUM N / COVALENT}

\section{CERAMICS}

Gary Fischman, Alfred University, (607) 871-2449 Richard M. Spriggs, Alfred University, (607) 871-2486

\section{- SYMPOSIUM O / FIBER-REINFORCED} CEMENTTTIOUS MATERIALS Sidney Mindess, University of British Columbia, (604) 228-6413

Jan P. Skalny, W.R. Grace \& Company, (301) 531-4597

\section{- SYMPOSIUM P / SCIENTIFIC BASIS} FOR NUCLEAR WASTE MANAGE-

\section{MENT XIV}

T. Abrajano, Jr., Argonne National Laboratory, (312) $972-4261$

Lawrence H. Johnson, Whiteshell Nuclear Research Establishment (204) 753-2311

\section{- SYMPOSIUM Q / HIGH-TEMPERATURE} ORDERED INTERMETALLIC ALLOYS James O. Stiegler, Oak Ridge National Laboratory, (615) $574-4065$

David P. Pope, University of Pennsytvania,

(215) $898-7246$

James C. Williams, GE Aircraft Engines, (513) 243-4531
- SYMPOSIUM R / NOVEL STRUCTURAL AND ELECTRONIC PROPERTIES OF POLYMERS

Joon Row, University of Cincinnati, (513) 556-3117 John M. Torkelson, Northwestern University, (312) $491-7449$ John Emerson, AT\&T Bell Laboratories, (609) 6392571

- SYMPOSIUM S / SYNTHESIS AND PROPERTIES OF NEW CATALYSTS: UTILIZATION OF NOVEL MATERIALS COMPONENTS AND SYNTHETIC TECHNIQUES

Marc J. Ledoux, Universite Louis Pasteur Strasbourg I, France

Edward W. Corcoran, Exxon Research and

Engineering, (201) 730-2465

Jack R. Knox, Knox Consulting Company, (312) $357-3707$

- SYMPOSIUM T / LONG-WAVELENGTH SEMICONDUCTOR MATERIALS Avishay Katz, AT\&T Bell Laboratories, (201) 582-2261 Robert M. Biefeld, Sandia National Laboratories, (505) 844-1556

R.J. Malik, AT\&T Bell Laboratories, (201) 582-6580 Robert L. Gunshor, Purdue University, (317) 4943509

- SYMPOSIUM U/ADVANCED TOMOGRAPHIC IMAGING METHODS FOR

THE STUDY OF MATERIALS

J.L. Ackerman, Massachusetts General Hospital (617) $726-3083$

W. Ellingson, Argonne National Laboratory, (312) $972-5068$

- SYMPOSIUM V / BIOMATERIALS (Chairs to be announced)

- SYMPOSIUM W I DYNAMICS OF DISORDERED SYSTEMS AND FRACTALS James P. Stokes, Exxon Research and Engineering, (201) $730-2426$

Mark O.Robbins, Johns Hopkins University, (301) $338-7204$

T.A. Witten, University of Chicago, (312) 702-0947; FAX (312) 702-5863

- SYMPOSIUM X/FRONTIERS OF MATERIALS SCIENCE

Rustum Roy, Pennsytvania State University, (814) 865-3421

- SYMPOSIUM Y / OAUNTUM STRUCTURES \& MICROLITHOGRAPHY T.P. Smith III, IBM T.J. Walson Research Center, (914) 945-2809

D. Kerns, IBM T.J. Watson Research Center (914) $945-1147$

S.D. Berger, AT\&T Bell Laboratories, (201) 582-2484 H. Craighead, Cornell University, (607) 255-2329

Meeting Chairs

Robert Hull, AT\&T Bell Laboratories, (201) 5826455

Gregory McCarthy, North Dakota State University (701) 237-7193

Frans Spaepen, Harvard University (617) 4953760 


\section{Booth \#209 at the MRS Exhibit in Boston}

The Physical and Mathematical Modelling of Tundish Operations

J. Szekely and $\mathbf{O}$.J. llgebusi

Reflecting the critical role of tundishes in affecting the quality of finished steel products. this book provides a good physical understanding of what is happening in tundishes and places tundish design into realistic perspective. It also addresses less well understood aspects and blends these considerations into a coherent approach to optimal design of tundish operations.

1989/111 pp./124 illus./11 tables/hardcover $\$ 54.00$

ISBN 0-387.96858-X

Materials Research and Engineering

\section{Ladle Metallurgy}

J. Szekely, G. Carlsson, and L. Helle

This text provides a comprehensive description of the science, practice, and economics of ladle metallurgical operations. The material draws heavily on previous SCABINJECT conferences and has been used tutorially by the authors. Topics covered include injection technology and metallurgy, injection practice in secondary metallurgy of steel, economic considerations, and the testing technique for powder injection.

$1989 / 166 \mathrm{pp} / 137$ illus./24 tables/hardcover $\$ 69.00$

ISBN 0-387-96798-2

Materials Research and Engineering

\section{Materials Testing for the Metal Forming Industry K. Pöhlandt}

This is the first comprehensive treatment of materials testing for the special demands of the metal forming industry. Testing methods for the forming behavior of metals as well as for metal forming tool materials are described. A list of ASTM, ISO and Euronorm standards are included for a complete reference source.

1989/226 pp./76 illus./hardcover $\$ 59.50$ (tent.)

ISBN 0-387-50651-9

\section{Modelling Hot Deformation of Steels \\ An Approach to Understanding and Behavior \\ Edited by J.G. Lenard}

Computer-aided modelling of microalloyed steels is examined in the context of controlled rolling. The book also reviews industrial practices and the difficulties associated with understanding high-temperature behavior. Also included is a promising new approach to deformation mapping.

1989/145 pp./47 illus./hardcover $\$ 49.50$

ISBN 0-387-50754-X

\section{Welding Metallurgy of Stainless Steel \\ E. Folkhard}

Approaches metallurgical reactions by discussing.constitution diagrams, solidification and segregation phenomena, phase transformations, precipitations of carbides and intermetallic phases, and cracking problems. In addition, it provides directions for welding techniques that avoid failure in practical welding operations.

$1988 / 279 \mathrm{pp} . / 115$ illus./hardcover $\$ 50.00$

ISBN 0-387-82043-4

\section{Non-Destructive Testing}

B. Hull and V. John

This introductory text treats the underlying principles and important applications of the major non-destructive inspection techniques now in use. In addition to complete chapter coverage of each technique, the text provides students and practicing engineers with a broad overview of the field. plus a review of recent developments.

$1988 / 144 \mathrm{pp} . /$ softcover $\$ 24.00$

ISBN 0-387-91325-4
Micromechanics and Inhomogeneity:

The Toshio Mura Anniversary Volume

Edited by G.J. Weng, M. Taya, and H. Abe

Written by leading researchers from the U.S., Japan, and Europe in honor of Mura's 65th birthday, the 37 original contributions span topics relevant to the applied mechanics and materials communities, but all linked by the indispensability of micromechanics to their understanding. Ample examples of analytical and experimental approaches with a special emphasis on composites and other new material systems are included.

1989/approx. $700 \mathrm{pp} / 225$ illus./hardcover $\$ 74.00$ (tent.) ISBN 0-387-97043-6

\section{Forthcoming - \\ Ultrasonic Testing of Materials \\ Fourth Edition \\ J. Krảutkrämer and H. Krautkrämer}

The established reference work in the field will be available in a fully revised fourth edition early in 1990 . It treats nondestrictive testing of solid materials using ultrasonic waves - from the basics up to the most sophisticated methods and including tips derived from 30 years of practical experience. This edition features improved coverage of physical fundamentals, updated reports on instrumentation, and new applications in the nuclear and space industries.

1990/approx. $700 \mathrm{pp} . / 564$ illus./hardcover $\$ 134.00$ (tent.)

ISBN 0-387-51231-4

\section{Principles of Magnetic Resonance \\ Third Edition}

C.P. Slichter

"...one of the best expositions of the quantum theory of resonance...It has my highest recommendation for use as a textbook." -J.O.S.A.

The third edition of this renowned graduate-level textbook offers new material in many areas, including new sections on one- and two-dimensional Fourier transforms, multiple quantum coherence, and magnetic resonance imaging.

1989/approx. $640 \mathrm{pp} . / 179$ illus./softcover $\$ 49.50$

ISBN 0-387-50157-6

Springer Series in Solid-State Sciences, Vol. I

\section{Also available -}

Electronic Structure and Optical Properties of

Semiconductors

Second Edition

M.L. Cohen and J.R. Chelikowsky

“...highlights some of the underlying mechanisms associated with optical processes in semiconductors and provides information related to their theoretical and experimental study...attention is directed toward techniques that investigate energy band structure..."

-CHOICE

$1989 / 264$ pp./161 illus./softcover $\$ 39.00$

ISBN 0-387-51391-4

Springer Series in Solid-State Sciences, Vol. 75

To order call Toll-Free: 1-800-SPRINGER (In NJ call 201-348-4033). Or FAX: $212-473-6272$. For mail orders please send payment plus $\$ 2.50$ for postage and handling to: Springer-Verlag New York, Inc., Attn.: K. Quinn, 175 Fifth Avenue, New York, NY 10010. We accept Visa, MC, AmEx, and Discover charges (with signature and expiration date noted) as well as personal checks and money orders. NY, NJ, and CA residents please add state sales tax.

\section{Springer-Verlag}

New York Berlin Heidelberg Vienna

London Paris Tokyo Hong Kong 